\title{
Refractory spontaneous hypoglycaemia: a diagnostic conundrum
}

\section{Seong Keat Cheah', David Halsall2, Peter Barker3 3 , John Grant'2, Abraham Mathews', Shyam Seshadri' and Singhan Krishnan'}

1Endocrinology Department, Hinchingbrooke Hospital, North West Anglia NHS Foundation Trust, Huntingdon, UK, 2Pathology Department, and ${ }^{3}$ Core Biochemical Assay Laboratory (CBAL), Addenbrooke's Hospital, Cambridge University Hospitals NHS Foundation Trust, Cambridge, UK
Correspondence should be addressed to S K Cheah

Email

seongkeatc@gmail.com

\section{Summary}

A frail 79-year-old lady with dementia presented with a 2-year history of frequent falls. Recurrent hypoglycaemic episodes were diagnosed and treated with continuous glucose infusion in multiple hospital admissions. Hypoadrenalism and hypothyroidism were ruled out. Whilst hypoglycaemic (blood glucose $1.6 \mathrm{mmol} / \mathrm{L}$ ), both plasma C-peptide and proinsulin concentrations, were inappropriately elevated at $4210 \mathrm{pmol} / \mathrm{L}(174-960)$ and $>200 \mathrm{pmol} / \mathrm{L}(0-7)$ respectively with plasma insulin suppressed at $12 \mathrm{pmol} / \mathrm{L}(0-180)$. Whilst reported cases of proinsulinoma are typically pancreatic in origin, radiological investigations of the pancreas in this patient did not identify abnormalities. Unexpectedly contrast CT identified a heterogeneously enhancing mass $(6.6 \mathrm{~cm})$ at the lower pole of the left kidney consistent with renal cell carcinoma. Non-islet cell tumour-induced hypoglycaemia has been associated with renal malignancy; however, a serum IGF2:IGF1 ratio measured at $<10$ effectively excludes this diagnosis. Concomitantly on the $\mathrm{CT}$, extensive peripherally enhancing heterogeneous mass lesions in the liver were identified, the largest measuring $12 \mathrm{~cm}$. A palliative approach was taken due to multiple comorbidities. On post-mortem, the kidney lesion was confirmed as clear cell renal carcinoma, whilst the liver lesions were identified as proinsulin-secreting neuroendocrine tumours. In conclusion, the diagnosis of proinsulinoma can be missed if plasma proinsulin concentration is not measured at the time of hypoglycaemia. In this case, the plasma insulin:C-peptide ratio was too high to be accounted for by the faster relative clearance of insulin and was due to proinsulin cross-reactivity in the C-peptide assay. In addition, the concomitant malignancy proved to be a challenging red herring.

\section{Learning points:}

- Even in non-diabetics, hypoglycaemia needs to be excluded in a setting of frequent falls. Insulin- or proinsulinsecreting tumours are potentially curable causes.

- Whilst investigating spontaneous hypoglycaemia, if plasma insulin concentration is appropriate for the hypoglycaemia, it is prudent to check proinsulin concentrations during the hypoglycaemic episode.

- Proinsulin cross-reacts variably with C-peptide and insulin assays; the effect is method dependent. In this case, the discrepancy between the insulin and C-peptide concentrations was too great to be accounted for by the faster relative clearance of insulin, raising the suspicion of assay interference. The C-peptide assay in question (Diasorin liaison) has been shown to be $100 \%$ cross reactive with proinsulin based on spiking studies with a proinsulin reference preparation.

- Whilst reported cases of proinsulinoma and $99 \%$ of insulinomas are of pancreatic origin, conventional imaging studies (CT, MRI or ultrasound) fail to detect neuroendocrine tumours $<1 \mathrm{~cm}$ in $50 \%$ of cases.

- The concomitant renal mass identified radiologically proved to be a red herring.

- In view of the rarity of proinsulinoma, no conclusive association with renal cell carcinoma can be established. \begin{tabular}{lll}
\hline open $(2)(1)$ & This work is licensed under a Creative Commons \\
Attribution-NonCommercial-NoDerivs 3.0
\end{tabular} Unported License. 


\section{Background}

Proinsulin-secreting neuroendocrine tumours are rarer and less well characterised than the more commonly identified insulinoma. A recent review reported proinsulinoma has a 2:1 female preponderance with mean age of diagnosis at 56 years (1). As to be expected, this lesion is typically found in the body and tail of the pancreas, which supports the diagnosis $(1,2,3)$, similar to insulinoma where $99 \%$ are pancreatic in origin (4). Localisation is challenging as conventional imaging modalities such as CT and MRI frequently miss small tumours (4). In addition, concomitant malignancy or extra-pancreatic lesions identified during localisation imaging may confound the diagnosis. We present a case report demonstrating this diagnostic challenge.

\section{Case presentation}

A frail 79-year-old lady with dementia presented with a 2-year history of frequent falls. These falls were not associated with loss of consciousness but preceded by dizzy spells. Due to a concomitant headache, neurological assessment attributed these episodes to migraine. She sustained a fractured right distal radius in February 2017 during one of the falls. She was found to be confused by the ambulance crew and her capillary blood glucose was noted to be $1.7 \mathrm{mmol} / \mathrm{L}$. This was treated with bolus intravenous dextrose solution, which was then administered as continuous infusion during her inpatient stay due to recurrent hypoglycaemia. She was treated for lower respiratory tract infection, which was thought to be the trigger for the hypoglycaemia given that the patient was not diabetic. She was discharged with advice for regular meals. She was readmitted with severe symptomatic hypoglycaemia the following day with a capillary blood glucose of $2.1 \mathrm{mmol} / \mathrm{L}$, which required an intravenous bolus of dextrose. Again, this was attributed to concurrent illness and general frailty despite the recurrent nature of the hypoglycaemia during her inpatient stay, and she was discharged.

A further admission the following day due to hypoglycaemia (capillary blood glucose, $1.7 \mathrm{mmol} / \mathrm{L}$ ) associated with loss of consciousness led to a series of investigations.

Despite dementia, a limited history obtained did not identify increased appetite. However, the patient had gained weight (50.1 kg in 2012 to $61.2 \mathrm{~kg}$ in 2017). Iatrogenic causes for her hypoglycaemia were thought unlikely as the patient had no access to insulin or oral hypoglycaemic agents and the patient lived in a nursing home. The patient had a 20 pack-year of smoking history but had not smoked for 20 years. There was no known family history of endocrine disease.

Duringherlatestadmission, shewashaemodynamically stable despite the recurrent hypoglycaemia. She did not manifest any signs of sepsis or infection and abdominal examination did not reveal any obvious masses.

\section{Investigations}

Table 1 summarised a series of investigations carried out. Hypoadrenalism and hypothyroidism were excluded, and the anterior pituitary profile was unremarkable.

During the hypoglycaemic episodes, insulin profile (Table 2) revealed an inappropriate elevation of plasma C-peptide and proinsulin; however, plasma insulin was not increased. The discrepant insulin and C-peptide ratio was due to the known cross-reactivity of the laboratory's C-peptide assay with proinsulin (Diasorin liaison). This assay has $\sim 100 \%$ cross-reactivity with proinsulin based on spiking studies with proinsulin reference material (Two previously analysed C-peptide samples were spiked with proinsulin international reference preparation IRR 09/296 such that the theoretical spiked proinsulin was approximately $40 \mathrm{pmol} / \mathrm{L}$.) Upon re-analysis of C-peptide in the samples post spike, the samples over-recovered 40 and $32 \mathrm{pmol} / \mathrm{L}$ of C-peptide compared to the original result (Supplementary report).

The biochemical profile was consistent with a proinsulin-secreting tumour. However, subsequent contrast CT of the abdomen and pelvis did not identify any pancreatic lesions (Fig. 1). Unexpectedly, a heterogeneously enhancing mass $(6.6 \mathrm{~cm})$ arising from the lower pole of the left kidney consistent with renal cell carcinoma was detected. Concomitantly, there were

Table 1 Endocrinological investigations.

\begin{tabular}{|c|c|c|}
\hline & Reference range & Values \\
\hline \multicolumn{3}{|l|}{ Cortisola, nmol/L } \\
\hline $0 \min$ & & 533 \\
\hline $30 \mathrm{~min}$ & $>450$ & 1079 \\
\hline ACTH, ng/L & $<50$ & 10 \\
\hline Free T3, pmol/L & $3.5-6.5$ & 3.6 \\
\hline Free T4, pmol/L & $10.0-19.8$ & 17.0 \\
\hline $\mathrm{TSH}, \mathrm{mU} / \mathrm{L}$ & $0.35-5.50$ & 1.24 \\
\hline $\mathrm{LH}, \mathrm{U} / \mathrm{L}$ & & 35.2 \\
\hline FSH, U/L & & 53.6 \\
\hline Prolactin, mU/L & $59-619$ & 247 \\
\hline $\mathrm{HbA} 1 \mathrm{c}, \mathrm{mmol} / \mathrm{mol}$ & $<42$ & 33 \\
\hline
\end{tabular}

${ }^{a}$ Cortisol taken as part of Short Synacthen Test. 
Table 2 Insulin profile.

\begin{tabular}{|c|c|c|c|}
\hline & Reference range & On admission & Repeat \\
\hline Glucose, mmol/L & & 3.50 & 1.57 \\
\hline C-Peptidea, pmol/L & $174-960$ & 5510 & 4210 \\
\hline Insulinc, pmol/L & $0-80$ & 25 & 12 \\
\hline Proinsulin' ${ }^{\mathrm{b}}, \mathrm{pmol} / \mathrm{L}$ & $0-7$ & $>200$ & $>200$ \\
\hline IGF1, nmol/L & $10-25$ & 8.1 & 5.7 \\
\hline IGF2, nmol/L & & & 24.5 \\
\hline IGF2:IGF1 & & & 4.3 \\
\hline
\end{tabular}

aC-peptide level performed using DiaSorin LIAISON C-Peptide assay (assay information accessible at http://assayinfo.diasorin.com/download/ IFUk_en_316171_09.pdf); bproinsulin level performed using in-house two site immunoassay method using antibody A6 from Agilent Dako, Stockport, UK, and an in-house anti-proinsulin antibody in accordance with the International Standard for human proinsulin NIBSC code: 09/296 instructions for use (Version 1.0; accessible at http://www.nibsc.org/ terms_and_conditions.aspx); cinsulin assayed using DiaSorinLIAISON Insulin assay - this assay has minimal cross-reactivity with proinsulin $(<1 \%$ @ $22680 \mathrm{pmol} / \mathrm{L}$ - data from manufacturer (http://assayinfo.diasorin.com/ download/IFUk_en_310360_05.pdf)).

extensive peripherally enhancing heterogeneous mass lesions in the liver, the largest measuring $12 \mathrm{~cm}$.

In view of the renal lesion, non-islet cell tumourinduced hypoglycaemia (NICTH) was suspected as this has been reported in cases of renal malignancies $(5,6)$. However, the IGF2:IGF1 ratio was measured at $<10$ (Table 2), which excludes this diagnosis (Assay provided by Guildford Royal Surrey County Hospital Peptide Hormone Laboratory (Supra-regional Assay Service accessible at http://www.sas-centre.org/assays/hormones/ insulin-like-growth-factor-ii-igf-ii).

\section{Treatment}

The hypoglycaemia episodes in this patient were difficult to treat. Regular intravenous hydrocortisone, titrating dose of diazoxide, intermittent glucagon and eventually subcutaneous octreotide were all tried and did not maintain euglycaemia, which required continuous intravenous dextrose infusion. Any attempt to discontinue the dextrose infusion immediately led to severe hypoglycaemia.

In view of multiple comorbidities and frailty, the patient was deemed to be an unsuitable candidate for surgical treatment, either for the renal cell carcinoma or a neuroendocrine tumour. The multiple liver lesions suggested metastatic disease associated with poor prognosis. After joint discussion between the patient, family members, palliative care team and the attending endocrinologists, a palliative approach was taken. With the knowledge that localisation of the insulin or

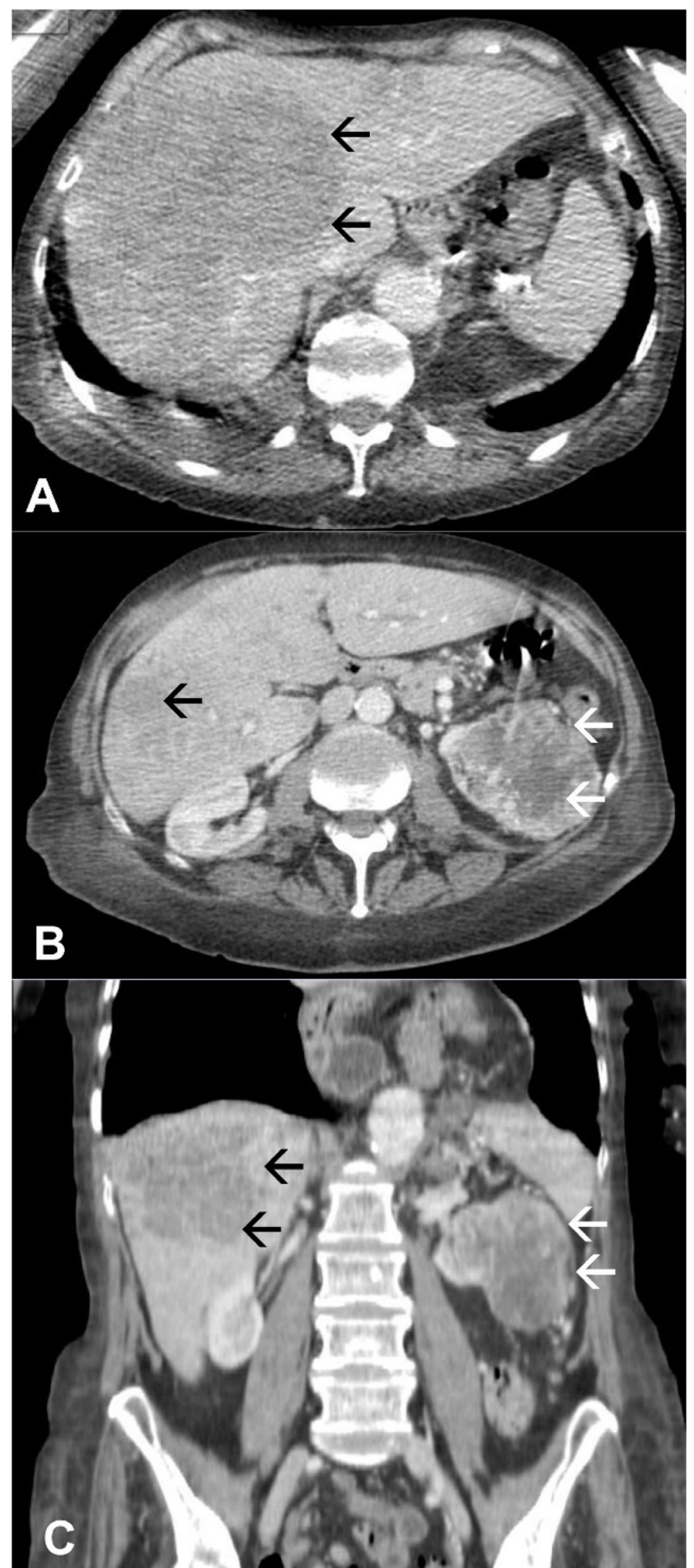

Figure 1

$C T$ abdomen and pelvis of transverse (A, B) and coronal (B) view

demonstrating the renal (white arrows) and liver lesions (black arrows) (C).

proinsulin-secreting tumour will not meaningfully alter the treatment outcome, more invasive imaging such as endoscopic ultrasound (EUS) or selective intraarterial 
calcium stimulation with digital subtraction angiography (DSA) were not justified. Dextrose infusions were discontinued, and patient died without obvious distress.

\section{Outcome and follow-up}

A post-mortem examination reported that the external surface of the pancreas was normal, and there are no palpable masses within. There was no mesenteric or retroperitoneal lymphadenopathy.

A friable tumour measuring of up to $60 \mathrm{~mm}$ with a large area of necrosis was identified at the lower pole of the left kidney. The microscopic findings of this lesion showed malignant cells with marked nuclear pleomorphism, widespread necrosis and focal invasion into blood vessels within the hilum. The immunological staining (Fig. 2) confirmed clear cell renal carcinoma with strong cytoplasmic staining for RCC, whilst insulin, chromogranin A and synaptophysin relevant for neuroendocrine tumour were negative.

The liver showed several tumours, the largest in the right lobe measured up to $110 \mathrm{~mm}$ with large areas of necrosis. The background liver was grossly normal. Microscopically, sections from the liver tumour showed infiltration of the parenchyma by centrally necrotic islands, and sheets of polygonal cells with striking nuclear pleomorphism and atypical mitotic figures. Tumour cells from the liver metastasis showed focal strong insulin immunoreactivity (Fig. 3), as well as widespread CD56, synaptophysin and chromogranin A staining, whilst negative for RCC. The morphology and immunoprofile of the liver tumours were most in keeping with metastases from a poorly differentiated proinsulin-secreting neuroendocrine tumour that was unlikely to be related to the renal cell carcinoma.

\section{Discussion}

This patient presented a 2-year history of frequent falls before her final admission. It was not clear if these falls preceded by dizziness were related to milder hypoglycaemic events in the past. Hypoglycaemia was documented in February 2017, which required three admissions within the same month. Her hypoglycaemia was initially attributed to lower respiratory infection despite the recurrent nature. An insulin profile taken earlier during admission would have expedited the final

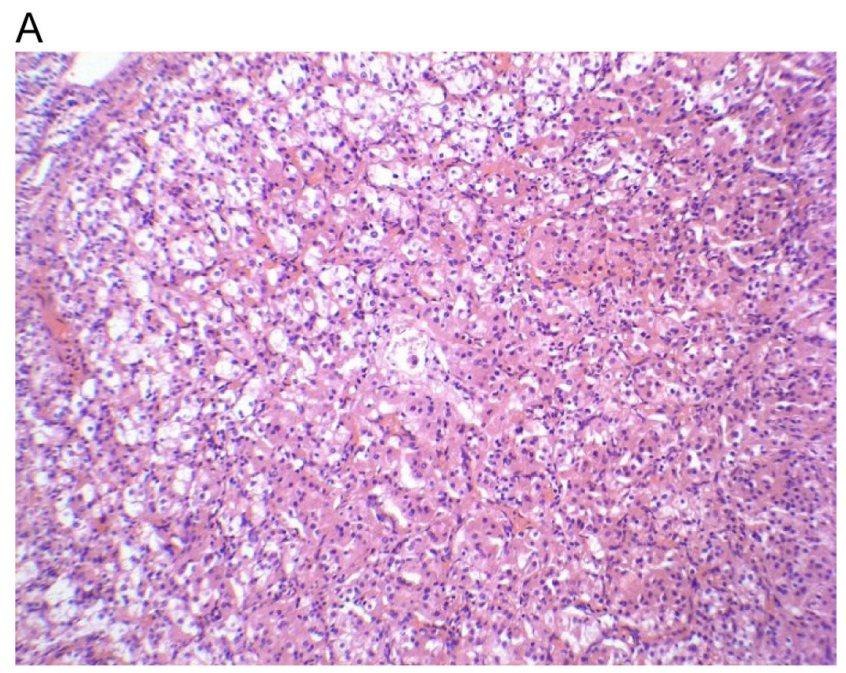

B
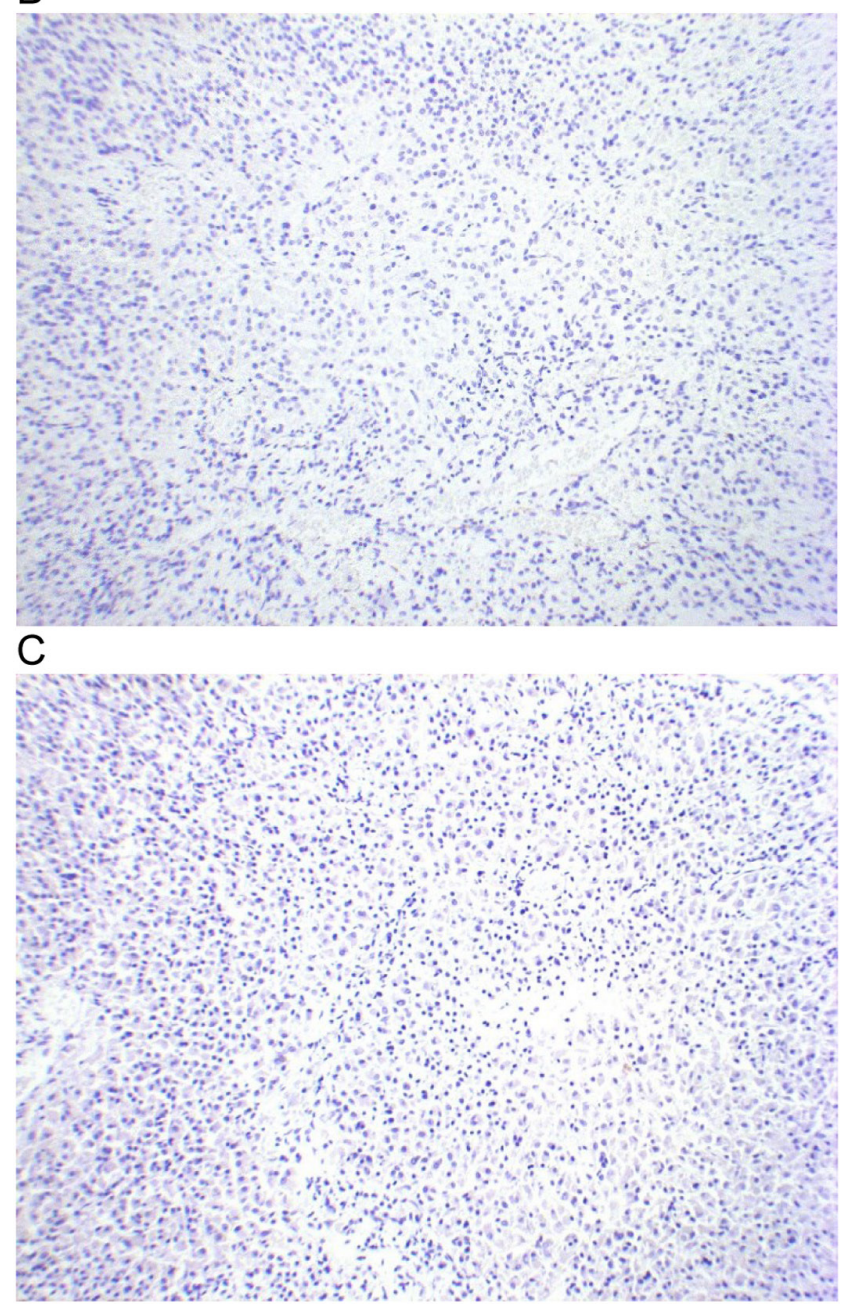

Figure 2

Kidney tumour. (A) H\&E staining (x10). (B) Synaptophysin staining $(\times 10)$. (C) Insulin immunoreactivity $(\times 10)$ 


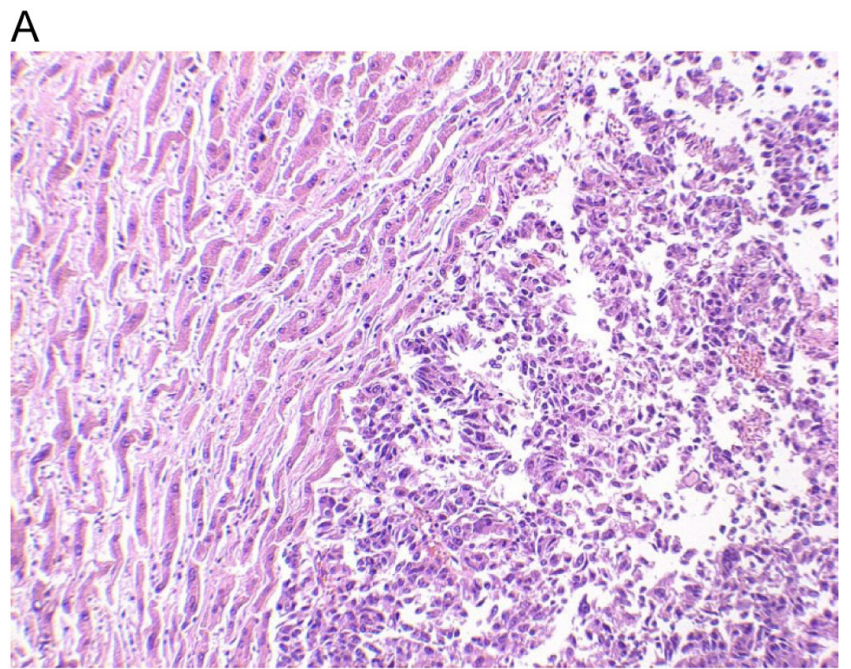

B
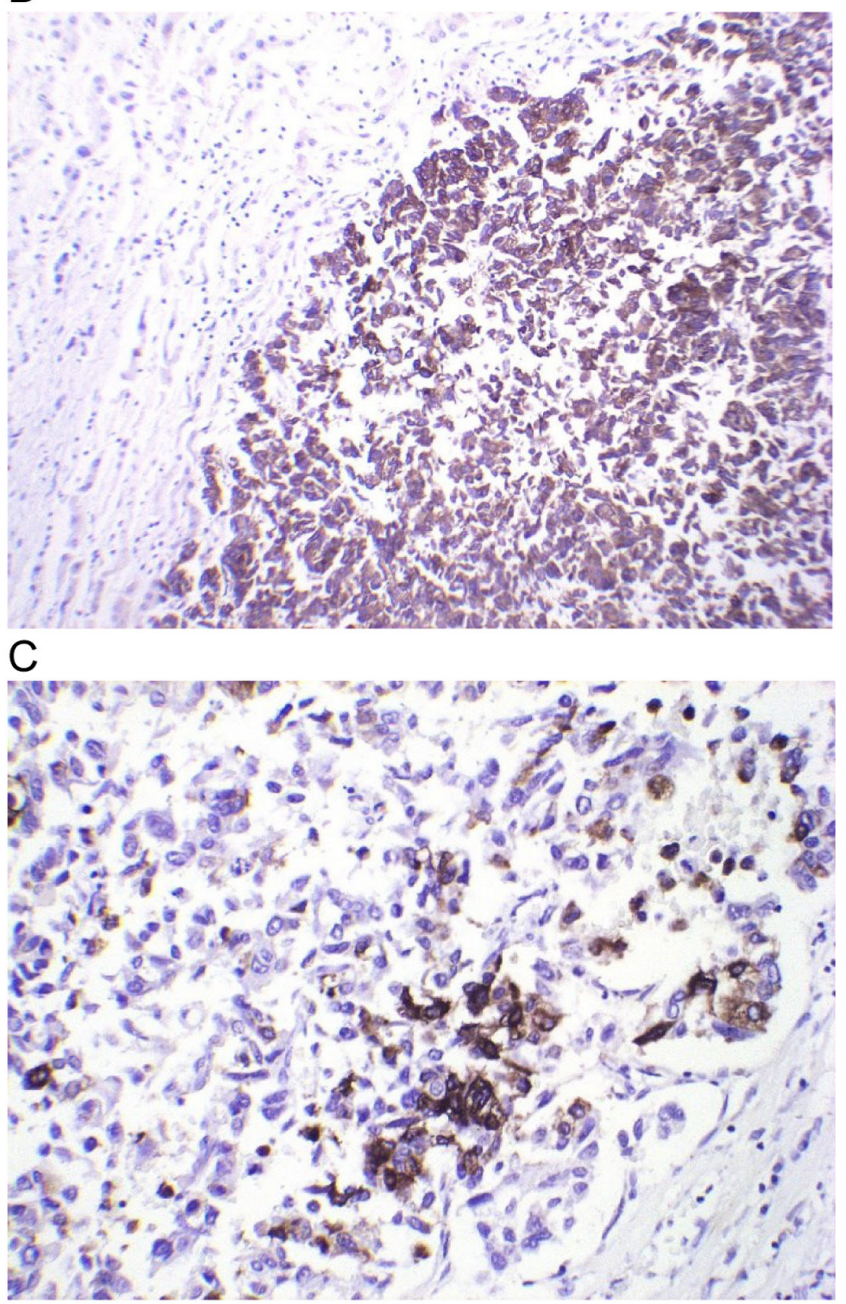

Figure 3

Liver tumour. (A) H\&E staining $(\times 10)$. (B) Synaptophysin staining $(\times 10)$. (C) Insulin immunoreactivity $(\times 20)$. diagnosis. However, it was understandably difficult to strike a balance between provision of meaningful treatment whilst avoiding over-investigation in a 79-yearold lady with established dementia. Her subsequent admissions with recurrent refractory hypoglycaemia prompted further investigation. This case report highlights the importance of considering hypoglycaemia in a setting of frequent falls in the elderly. With hindsight, if an association between her falls and hypoglycaemia had been made earlier, the patient may have benefited potentially curative treatment for proinsulinoma before developing extensive liver metastasis.

Proinsulinoma is exceedingly rare, and hence, it is not as well described as its more commonly known counterpart, insulinoma. A systemic review of 16 proinsulinoma cases reported a 2:1 female preponderance with mean age of diagnosis at 56 years (1). The majority of these cases presented with symptomatic hypoglycaemia with normal or low insulin levels. The systemic review reported median tumour size of $1.2 \mathrm{~cm}$ and normalisation of hormonal levels post resection without recurrence. As to be expected, the lesion is usually identified in the body or tail of the pancreas, which supports the diagnosis $(1,2,3)$, similar to insulinoma where $99 \%$ are pancreatic (4). Therefore, if insulin concentrations are found to be appropriate whilst investigating spontaneous hypoglycaemia (glucose $<3 \mathrm{mmol} / \mathrm{L}$ ) (7), it is prudent to measure plasma proinsulin as the diagnosis of a proinsulinsecreting neuroendocrine tumour can be missed.

The prohormone proinsulin consists of 86 amino acids including the insulin A and B chains linked by C-peptide. Following cleavage of proinsulin, intact C-peptide and insulin are secreted in equimolar amounts (8). Proinsulin is not removed by liver, it has a half-life three to four times that of insulin, therefore, proinsulin can accumulate in the blood and account for $12-20 \%$ of the circulating immunoreactive insulin in the basal state. Human proinsulin has about $7-8 \%$ of the biological activity of insulin and is mainly degraded by kidney (9). Despite the equimolar secretion of insulin and C-peptide, insulin has a relatively faster clearance compared to C-peptide. Proinsulin often cross-reacts variably with C-peptide and insulin assays depending on the immunoassays adopted by local laboratory.

In this case, the discrepancy between the insulin and C-peptide concentrations was too great to be accounted for by the faster clearance of insulin, raising the suspicion of assay interference. The Diasorin liaison 
C-peptide assay used here has been shown to be $100 \%$ cross reactive with proinsulin, based on spiking studies with a proinsulin reference preparation (Supplementary report); this is reflected in the manufacturer's instructions for use. Insulin assays also have the potential for variable cross-reactivity with proinsulin so the importance of familiarity with local assays cannot be overemphasised as neoplastic production of peptide hormones may generate unusual isoforms or poorly processed peptide fragments, which have the potential to confound immunoassay methods. Unfortunately, material was not available to compare results from alternate assays with different crossreactivities or to use definitive methods such as tandem mass-spectrometry.

UK and Ireland Neuroendocrine Tumour Society guidelines suggested that for localising the primary pancreatic neuroendocrine tumour a multimodality approach is best, including CT, MRI and EUS, often together with somatostatin receptor scintigraphy (SSRS) and in some centres, selective intraarterial calcium stimulation with DSA (10). This patient only underwent contrast CT and was too frail to proceed with other modalities for localisation. Whilst reported cases of proinsulinoma and 99\% of insulinoma are pancreatic in origin, conventional imaging studies (CT, MRI or ultrasound) fail to detect neuroendocrine tumours $<1 \mathrm{~cm}$ in $50 \%$ of cases (4). Despite unremarkable pancreas in contrast CT examination in this patient, the multiple liver lesions were confirmed to be proinsulin-secreting neuroendocrine tumours, raising the possibility of these were metastatic lesions from a small volume primary in pancreas not evident on CT. Ideally, EUS or selective intraarterial calcium stimulation with DSA would have been helpful in localisation after CT. EUS reported sensitivity of $79-100 \%$, with the advantage to allow tissue sampling and assessment of local invasion of the tumour to decide on surgical approach (enucleation or Whipple's procedure). Interestingly, SSRS only reported sensitivity of $50-60 \%$ in detection of insulinomas, as opposed to $75 \%$ sensitivity in detection of other types of pancreatic neuroendocrine tumours (10).

In addition to the challenges caused by the C-peptide assay interference, and investigative limitations due to patient's frailty, the detection of the unexpected renal mass and the known association of NICTH with renal malignancy along with the relatively low plasma insulin raised suspicion of NICTH as a possible diagnosis $(5,6)$. The commonest cause of NICTH is the overproduction and IGF2. The IGFs are structurally similar to proinsulin and mimic the biochemical effects of insulin upon isolated tissues in vitro, and hence, suppression of insulin, glucagon and growth hormone secretion were observed. Although fibromas and fibrosarcoma are best known causes of NICTH, no histological type of tumour can be excluded from causing NICTH due to lack of large studies (11). Disproportionately high IGF2:IGF1 ratios are suggested as a pathognomonic feature of NICTH (12). In this lady, the IGF2:IG1 ratio $<10$ sampled during hypoglycaemia ruled out NICTH. In view of the rarity proinsulinoma, no conclusive association with renal cell carcinoma can be established.

\section{Supplementary data}

This is linked to the online version of the paper at https://doi.org/10.1530/ EDM-18-0049.

\section{Declaration of interest}

The authors declare that there is no conflict of interest that could be perceived as prejudicing the impartiality of the research reported.

\section{Funding}

This research did not receive any specific grant from any funding agency in the public, commercial or not-for-profit sector.

\section{Patient consent}

The patient is deceased. No identifying information has been revealed and anonymity is maintained.

\section{Author contribution statement}

Seong Keat Cheah (Specialty Registrar, Endocrinology) reviewed and managed the patient during her inpatient stay under the supervision of $\mathrm{Dr}$ Shyam Seshadri; request and follow-up of key investigations for the case and wrote the case report. David Halsall (Biochemist) provided information regarding C-peptide assay and key suggestions on interpretation of insulin profile and reviewed the case report. Peter Barker (Biochemist) performed cross-reactivity studies on C-peptide assay. John Grant (Pathology Consultant) performed post-mortem assessment. Abraham Mathew (Senior House Officer) reviewed the patient and supported clinical management. Shyam Seshadri (Endocrinology Consultant) is the patient's named physician during her inpatient stay in ward, who was directly involved in her inpatient management. Singhan Krishnan (Endocrinology Consultant) provided key clinical suggestions for the management of the case and reviewed the case report.

\section{References}

1 Murtha TD, Lupsa BC, Majumdar S, Jain D \& Salem RR. A systematic review of proinsulin-secreting pancreatic neuroendocrine tumors. Journal of Gastrointestinal Surgery 201721 1335-1341. (https://doi. org/10.1007/s11605-017-3428-8)

2 Fadini GP, Maran A, Valerio A, Meduri F, Pelizzo M, Miotto D, Lanza C, Altavilla G \& Avogaro A. Hypoglycemic syndrome in a patient with proinsulin-only secreting pancreatic adenoma (proinsulinoma). Case Reports in Medicine 20112011 1-5. 
3 Pérez-Pevida B, Idoate MÁ, Fernández-Landázuri S, Varo N \& Escalada J. Hypoglycemic syndrome without hyperinsulinemia. A diagnostic challenge. Endocrine Pathology 201627 50-54. (https://doi. org/10.1007/s12022-016-9415-x)

4 Jensen RT, Cadiot G, Brandi ML, De Herder WW, Kaltsas G, Komminoth P, Scoazec JY, Salazar R, Sauvanet A, Kianmanesh R, et al. ENETS Consensus Guidelines for the management of patients with digestive neuroendocrine neoplasms: functional pancreatic endocrine tumor syndromes. Neuroendocrinology 201295 98-119. (https://doi.org/10.1159/000335591)

5 de Groot JWB, Rikhof B, van Doorn J, Bilo HJG, Alleman MA, Honkoop AH \& van der Graaf WT. Non-islet cell tumour-induced hypoglycaemia: a review of the literature including two new cases. Endocrine-Related Cancer 200714 979-993. (https://doi.org/10.1677/ ERC-07-0161)

6 Fernando HS, Hawkyard SJ, Poon P \& Musa M. Renal cell carcinoma with non-islet cell tumor hypoglycemia. International Journal of Urology 200613 985-986. (https://doi.org/10.1111/j.14422042.2006.01452.x)

7 Cryer PE, Axelrod L, Grossman AB, Heller SR, Montori VM, Seaquist ER \& John Service F. Evaluation and management of adult hypoglycemic disorders: an Endocrine Society Clinical Practice Guideline Summary of Recommendations. Journal of Clinical Endocrinology and Metabolism 200994 709-728. (https://doi. org/10.1210/jc.2008-1410)

8 Steiner DF. The proinsulin C-peptide - a multirole model. Experimental Diabesity Research 20045 7-14. (https://doi. org/10.1080/15438600490424389)

9 Gardner DG, Shoback DM, Greenspan FS \& Francis S. Greenspan's Basic and Clinical Endocrinology, p 880. McGraw-Hill Medical, 2011.

10 Ramage JK, Ahmed A, Ardill J, Bax N, Breen DJ, Caplin ME, Corrie P, Davar J, Davies AH, Lewington V, et al. Guidelines for the management of gastroenteropancreatic neuroendocrine (including carcinoid) tumours (NETs). Gut 201261 6-32. (https://doi. org/10.1136/gutjnl-2011-300831)

11 Marks V \& Teale JD. Tumours producing hypoglycaemia. Endocrine-Related Cancer 19985 111-129. (https://doi.org/10.1677/ erc.0.0050111)

12 Teale JD \& Marks V. Inappropriately elevated plasma insulin-like growth factor II in relation to suppressed insulin-like growth factor I in the diagnosis of non-islet cell tumour hypoglycaemia. Clinical Endocrinology 199033 87-98.

Received in final form 18 May 2018

Accepted 15 June 2018 